\title{
Dielectric Relaxation in Dimethyl Sulfoxide/Water Mixtures Studied by Microwave Dielectric Relaxation Spectroscopy
}

\author{
Zijie Lu, ${ }^{\dagger}$ Evangelos Manias, ${ }^{\ddagger}$ Digby D. Macdonald, ${ }^{*}$, and Michael Lanagan $^{\S}$ \\ Center for Electrochemical Science and Technology, Polymer Nanostructures Laboratory, Department of \\ Materials Science and Engineering, and Center for Dielectric Studies, Materials Research Laboratory, The \\ Pennsylvania State University, University Park, Pennsylvania 16802
}

Received: June 24, 2009; Revised Manuscript Received: August 17, 2009

\begin{abstract}
Dielectric spectra of dimethyl sulfoxide (DMSO)/water mixtures, over the entire concentration range, have been measured using the transmission line method at frequencies from $45 \mathrm{MHz}$ to $26 \mathrm{GHz}$ and at temperatures of 298-318 K. The relaxation times of the mixtures show a maximum at an intermediate molar fraction of DMSO. The specific structure of mixtures in different concentration regions was determined by the dielectric relaxation dynamics, obtained from the effect of temperature on the relaxation time. A water structure "breaking effect" is observed in dilute aqueous solutions. The average number of hydrogen bonds per water molecule in these mixtures is found to be reduced compared to pure water. The increase in the dielectric relaxation time in DMSO/water mixtures is attributed to the spatial (steric) constraints of DMSO molecules on the hydrogen-bond network, rather than being due to hydrophobic hydration of the methyl groups. The interaction between water and DMSO by hydrogen bonding reaches a maximum at a DMSO molar fraction of 0.33, reflected by the maximum activation enthalpy for dielectric relaxation in this concentration, suggesting the formation of a stoichiometric compound, $\mathrm{H}_{2} \mathrm{O}-\mathrm{DMSO}-\mathrm{H}_{2} \mathrm{O}$. In highly concentrated solutions, negative activation entropies are observed, indicating the presence of aggregates of DMSO molecules. A distinct antiparallel arrangement of dipoles is obtained for neat DMSO in the liquid state according to the Kirkwood correlation factor $\left(g_{\mathrm{K}}=0.5\right)$, calculated from the static permittivity. The similarity of the dielectric behavior of pure DMSO and DMSO-rich mixtures suggests that dipole-dipole interactions contribute significantly to the rotational relaxation process in these solutions.
\end{abstract}

\section{Introduction}

Water is a highly self-associated liquid with an open, low coordination number structure; the coordination number is about 4.8 at $20{ }^{\circ} \mathrm{C} .{ }^{1} \mathrm{~A}$ number of models have been proposed to account for the physical properties of water., ${ }^{2,3}$ Nevertheless, water remains an anomalous liquid where no single model is able to explain all of its properties. ${ }^{4}$ Ionic, polar, and hydrophobic solutes perturb the structure of water in different ways with profound consequences on their solubility, hydration thermodynamics, and their association with other solutes. Soluteinduced perturbations in the water structure are, in turn, less understood than the structure of water itself and have long been a subject of controversy in chemistry, biology, and physiology. ${ }^{5}$

In this paper, we present and discuss results of a dielectric relaxation study of aqueous solutions of dimethyl sulfoxide (DMSO). The interest in DMSO is due, in part, to the wide use of DMSO $-\mathrm{H}_{2} \mathrm{O}$ as solvents and reaction media. DMSO is a polyfunctional molecule with a highly polar $\mathrm{S}=\mathrm{O}$ group and two hydrophobic $\mathrm{CH}_{3}$ groups. The partial negative charge on the oxygen atom of DMSO molecule favors the formation of the hydrogen bonds with water molecules, whereas the nonpolar $\mathrm{CH}_{3}$ groups may give rise to hydrophobic hydration and hydrophobic association of DMSO molecules. Many observa-

* Corresponding author. E-mail: ddm2@ psu.edu. Tel.: (814) 863-7772. Fax: (814) 863-4718.

Center for Electrochemical Science and Technology, Department of Materials Science and Engineering.

₹ Polymer Nanostructures Laboratory, Department of Materials Science and Engineering.

${ }^{\S}$ Center for Dielectric Studies. tions ${ }^{6-8}$ suggest that DMSO forms hydrogen bonds with water molecules throughout the whole composition range. Studies of the thermodynamic ${ }^{9,10}$ and transport properties ${ }^{11,12}$ of the DMSO/ water system resulted in the generally accepted conclusion that in the DMSO mole fraction range of $0.3-0.4$ DMSO interactions with water, due to hydrogen bonding, are at a maximum.

The influence of DMSO on the molecular structure of liquid water has been studied by many authors, and a number of different and often mutually exclusive explanations exist in literature. The $\mathrm{HH}$ (water-H to water-H), MM (methyl-H to methyl-H), and the MH (methyl-H to water-H) pair correlation functions in DMSO/water mixtures have been measured by Luzar and co-workers ${ }^{13-16}$ with neutron diffraction. The water structure is found to be weakly affected by the presence of DMSO, but the percentage of water molecules that are hydrogen-bonded to each other is substantially reduced compared to pure water. Furthermore, no evidence for hydrophobic association of DMSO molecules has been observed in DMSO/ water mixtures, which is similar to previous findings from IR spectroscopy of the $\mathrm{OH}$ and OD bands ${ }^{10,17}$ and other experiments. ${ }^{18}$ These results point to a "breaking" of water structure caused by the presence of DMSO molecules. Many other experiments, including temperature of maximum density measurements ${ }^{19}$ and ionic conductance studies, ${ }^{20}$ also lead to the conclusion that small amounts of DMSO acts as a structure breaker in water. On the other hand, neutron inelastic scattering and X-ray diffraction studies ${ }^{7}$ and NMR measurements ${ }^{6,21}$ indicate that small amounts of DMSO increase the molecular order of water. Infrared spectroscopy ${ }^{17}$ as well as density and 
partial molar volume measurements ${ }^{22}$ show that small quantities of DMSO have little effect on the water hydrogen bonding. Computer simulations of the structure of DMSO/water mixtures have also been discussed on the basis of radial distribution functions. ${ }^{15,23-31}$ Typical molecular configurations, including DMSO $\cdot 2 \mathrm{H}_{2} \mathrm{O}$ clusters, have been revealed in the simulations. DMSO was found to "enhance" the structure of water in very dilute solutions, whereas a further increase in DMSO concentration in the solution led to a "breakdown" of the water structure.

Dielectric relaxation spectroscopy (DRS) probes the response of the total dipole moment of a system, $M(t)=\sum \mu_{j}(t)$, to a time-dependent external electrical field. ${ }^{32}$ This inherent ability to monitor the cooperative motion of a molecular ensemble makes it a powerful tool for investigating liquids whose structure and dynamics are dominated by intermolecular hydrogen bonds. Dielectric relaxation studies of DMSO/water mixtures have been the subject of several investigations. ${ }^{33-37}$ Kaatze et al. ${ }^{33}$ measured the dielectric spectra of the aqueous DMSO solutions in the whole composition range at $25^{\circ} \mathrm{C}$ between $1 \mathrm{MHz}$ and $40 \mathrm{GHz}$. A strong increase of relaxation time with increasing DMSO concentration was observed for the mixtures in the water-rich region. Similar behavior was also observed by Lyashchenko et al. ${ }^{34}$ and Puranik et al. ${ }^{36}$ This increase of relaxation time was explained to be due to the hydrophobic hydration effects simply as an analogy with the increasing dielectric relaxation times usually found around macromolecules. However, this analogy ignores the effects of strong H-bonds between water and DMSO molecules and may not be always correct. Buchner et al. ${ }^{38}$ applied the absolute reaction rate theory to determine the dielectric activation enthalpy and entropy for water from the dielectric relaxation times and to understand the molecular dynamics in water. The similar approach has been used in the investigation of alcohol/water systems. ${ }^{39}$ The same method seems to be applicable to the DMSO/water mixtures. Therefore, a main purpose of this paper is to investigate the dielectric relaxation in the DMSO/water mixtures in hope of understanding the dielectric mechanism of the system.

It is also interesting to investigate dielectric relaxation of the highly concentrated DMSO solutions. Dimethyl sulfoxide is a self-associated liquid, reflected by its high melting point (18.55 ${ }^{\circ} \mathrm{C}$ ), boiling point $\left(189.0{ }^{\circ} \mathrm{C}\right)$, and high molar entropy of vaporization $\left(124 \mathrm{~J} \cdot \mathrm{K}^{-1} \cdot \mathrm{mol}^{-1}\right)$. Infrared and Raman studies ${ }^{40-42}$ suggest the existence of dimers and higher polymers in liquid DMSO. A distinct antiparallel ordering of the molecular dipoles has been observed by static permittivity measurements ${ }^{33}$ and by molecular dynamics simulation. ${ }^{23}$ In highly concentrated solutions, insufficient water molecules are available to completely hydrate the DMSO molecules. One would expect that it is solute-solute association that leads to the formation of microphases in these solutions. But, one can also imagine that molecular complexes are formed by hydrogen bonding between DMSO and water molecules. For example, in DMSO-rich mixtures, a recent molecular dynamics (MD) simulation ${ }^{29,30}$ revealed the existence of $1 \mathrm{H}_{2} \mathrm{O}-2 \mathrm{DMSO}$ cluster (an association of a pair of DMSO molecules through their oxygen atoms linked by one water molecule).

In view of the inconclusive results of previous studies concerning the structure of DMSO/water mixtures, it is appropriate to investigate the temperature dependence of the dielectric spectra of aqueous DMSO solutions over the whole composition range. Accordingly, in this study, we measured the dielectric relaxation spectra over a wide frequency range of 45 $\mathrm{MHz}$ to $26 \mathrm{GHz}$ and at temperatures ranging from 25 to $45^{\circ} \mathrm{C}$. The aim of this paper is to explore the relative contributions of the hydrophobic and hydrophilic groups of DMSO to modifying water structure and to elucidate the structure of DMSO/water mixtures over the whole concentration range.

\section{Experimental Section}

Dimethyl sulfoxide (Sigma-Aldrich, 99.97\%) was used as received. Double-distilled and deionized water (>18 $\mathrm{M} \Omega$, Millipore) was used in all the experiments. The DMSO/water mixtures at different DMSO mole fractions ( $x_{\mathrm{DMSO}}$ ) were prepared by mixing appropriate amounts of solute and solvent.

The complex permittivity spectra of the DMSO/water mixtures in the frequency range from $45 \mathrm{MHz}$ to $26 \mathrm{GHz}$ were measured with a transmission line using a traveling-wave method. The wave transmitted through a liquid-filled coaxial line in the form of transmitted $S$-parameter $\left(S_{21}\right)$ was measured by an HP8510C vector network analyzer. This technique was previously developed to measure the dielectric properties of polymer electrolyte membranes in the authors' laboratory. ${ }^{43,44}$ In this work, the DMSO/water mixture sample was placed at the end of a vertically positioned coaxial line of $7 \mathrm{~mm}$ o.d. and $10 \mathrm{~cm}$ long. A thin layer of dielectric plug (vacuum grease in this work), whose dielectric properties are close to that of air, was placed in the probe connector to prevent liquid leakage. The influence of the dielectric plug on the measurement has been shown ${ }^{44}$ to be negligible and was not taken into account in the solving for dielectric properties. Before measurement, the system was calibrated with an open, a short, and a known load (50 $\Omega$ ). Calibration of $S_{21}$ magnitude was made by performing a simple through connection with air as the dielectric medium. Due to the high dielectric loss of the DMSO/water mixtures, several sample lengths, varying from 10 to $1 \mathrm{~cm}$, were used to obtain the optimal measurement of the dielectric spectra. Detailed explanation for the apparatus, the measurement procedure, and the accuracy of the data as well as the validation of the technique for liquid media have been reported and discussed in ref 44 .

DRS measurements were carried out at temperatures over the range of $25-45{ }^{\circ} \mathrm{C}$ at intervals of $5{ }^{\circ} \mathrm{C}$. The coaxial line was heated by using a heating tape, and the temperature was controlled by a temperature controller to a precision of \pm 0.5 ${ }^{\circ} \mathrm{C}$. The uncertainties of the complex permittivity values depend on the accuracy of the scattering parameters and on the magnitude of the $\varepsilon^{\prime}$ and $\varepsilon^{\prime \prime}$, themselves. Typical errors of less than $3 \%$ for $\varepsilon^{\prime}$ and $\varepsilon^{\prime \prime}$ were obtained.

The experimental dielectric spectra were fitted in a complex nonlinear least-squares routine by using various models represented by the Havriliak-Negami function ${ }^{45}$

$$
\varepsilon(\omega)=\varepsilon_{\infty}+\frac{\varepsilon_{\mathrm{s}}-\varepsilon_{\infty}}{\left[1+(j \omega \tau)^{1-\alpha}\right]^{\beta}}
$$

where $\varepsilon_{\mathrm{s}}$ is the static permittivity, $\varepsilon_{\infty}$ is the high-frequency limiting permittivity, and $\tau$ is the relaxation time. $\alpha(0 \leq \alpha \leq$ $1)$ and $\beta(0 \leq \beta \leq 1)$ are shape parameters describing symmetric and asymmetric distribution of relaxation times, respectively. Three well-known models are limiting cases of this general equation; they are the Debye equation $(\alpha=0, \beta=1)$, the Cole-Cole eq $(0 \leq \alpha<1, \beta=1)$, and the Davidson-Cole equation $(\alpha=0,0<\beta \leq 1)$.

\section{Results}

Typical examples of the permittivity spectra of the DMSO/ water mixtures in the whole composition range and at temper- 


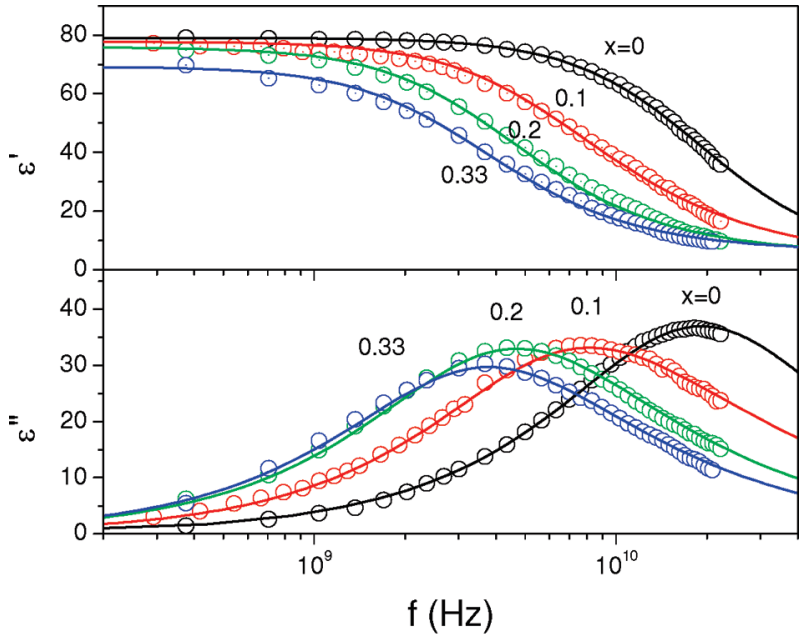

(a)

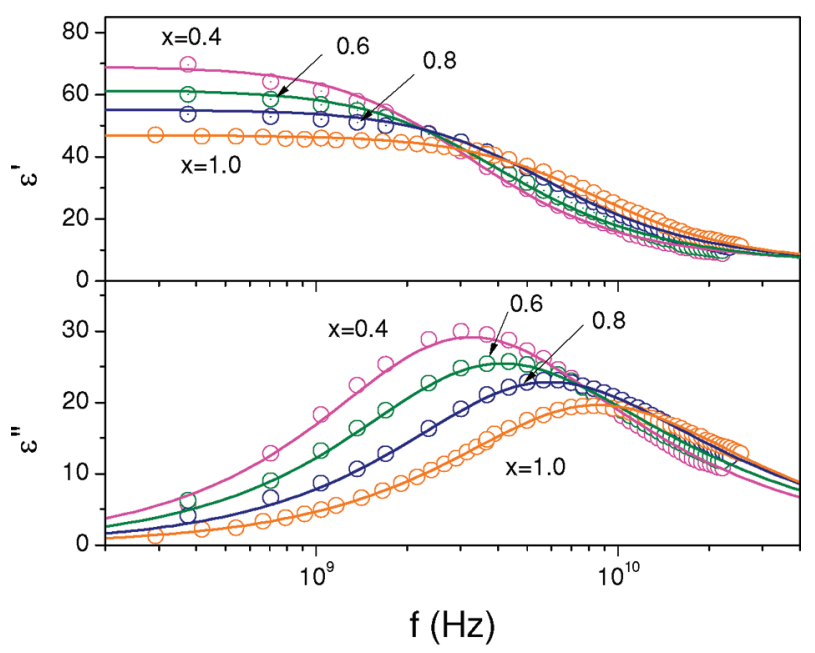

(b)

Figure 1. Complex permittivity spectra $\left(\varepsilon^{\prime}\right.$ and $\left.\varepsilon^{\prime \prime}\right)$ of DMSO/water mixtures at $25{ }^{\circ} \mathrm{C}$ at concentrations of (a) $0 \leq x_{\text {DMSO }} \leq 0.33$ and (b) $0.4 \leq x_{\text {DMSO }} \leq 1$. The numbers in the figure represent the mole fraction of DMSO. The solid lines are the best fit with a Davidson-Cole function.

atures of $25-45{ }^{\circ} \mathrm{C}(298-318 \mathrm{~K})$ are shown in Figures 1 and 2 , where Figure 1 shows the permittivity spectra of the mixtures over the entire concentration range at $298 \mathrm{~K}$ and Figure 2 shows the spectra of the mixture of $x_{\mathrm{DMSO}}=0.15$ at 298,308 , and $318 \mathrm{~K}$. The plot in Figure $2 \mathrm{~b}$ is commonly called a Cole-Cole plot. The dielectric spectrum of pure water in this frequency range is usually described as being a Debye relaxation. ${ }^{46-49}$ The relaxation times and static permittivity obtained by fitting the experimental data with a single Debye relaxation model are in accordance with the literature data ${ }^{46-49}$ to within $\pm 0.5 \%$. The permittivity spectra of pure DMSO are best fit by a DavidsonCole function $(\alpha=0,0<\beta \leq 1$ in eq 1$){ }^{33,50}$ The dielectric parameters of DMSO obtained in this work are in good agreement with the literature data. ${ }^{33,50}$ The accurate measurements of the dielectric spectra of pure water and pure DMSO offer further validation of the transmission line technique used in this work.

The complex plane representation (i.e., Cole-Cole plot) of the dielectric spectra of the DMSO/water mixtures is a distorted curve toward the real $\left(\varepsilon^{\prime}\right)$ axis (see Figure $2 b$ ), indicating that there is a distribution of relaxation times in these systems. The permittivity spectra, $\varepsilon^{*}(\omega)$, of the mixtures at various composi-

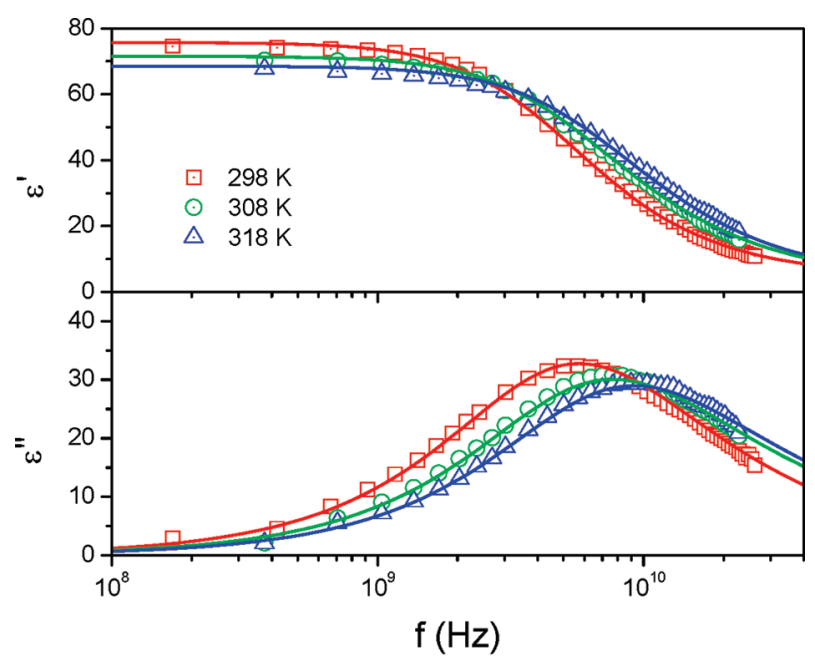

(a)

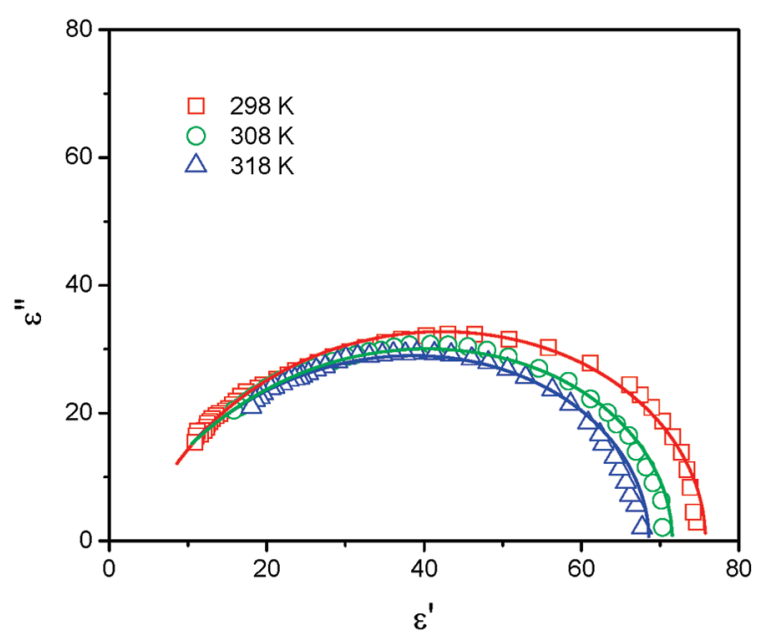

(b)

Figure 2. (a) Complex permittivity spectra of a DMSO/water mixture with the concentration of $x_{\text {DMSO }}=0.15$ at 298, 308, and $318 \mathrm{~K}$; (b) Cole-Cole diagram of $\varepsilon^{\prime}$ and $\varepsilon^{\prime \prime}$. Open symbols represent experimental data. The solid lines are calculated from the Davidson-Cole relaxation spectral function.

tions were then analyzed by simultaneously fitting $\varepsilon^{\prime}(\omega)$ and $\varepsilon^{\prime \prime}(\omega)$ to eq 1 with adjustable $\alpha$ and $\beta$. The best results, i.e., the minimum variance of the fit and a consistent set of relaxation parameters as a function of composition and temperature, are obtained using the Davidson-Cole function ( $\alpha=0$ in eq 1). This is in agreement with the literature..$^{33,36}$ In order to check more complicated spectral functions that might be related to more specific solution models, as shown in the case of diethyl sulfoxide/water mixtures, ${ }^{51}$ we also attempted to fit $\varepsilon^{*}(\omega)$ by a sum of multiple dispersion steps, where for each relaxation process a band shape defined by Debye, Cole-Cole, DavidsonCole, or Havriliak-Negami function can be selected. However, for DMSO/water mixtures, even a sum of two simple Debye relaxation processes, assuming a discrete contribution from water and pure DMSO, has been proven to be invalid. This was also pointed out by Kaatze et al. ${ }^{33}$ The authors are aware that the use of Davidson-Cole spectral function is less theoretically based than the functions like Debye. However, recognizing a previous fitting procedure described in the literature, ${ }^{33,36}$ we believe that the Davidson-Cole function is the most appropriate way for displaying the frequency dispersion of the data. 
TABLE 1: Dielectric Parameters of the Davidson-Cole Relaxation Spectral Function (Eq 1 with $\alpha \equiv 0)$ for DMSO/Water Mixtures

\begin{tabular}{|c|c|c|c|c|c|c|c|c|c|}
\hline & $\varepsilon_{\mathrm{s}}(\leq \pm 0.4)$ & $\tau(\mathrm{ps})(\leq \pm 0.3)$ & $\beta(\leq \pm 0.01)$ & $\varepsilon_{\infty}(\leq \pm 0.2)$ & & $\varepsilon_{\mathrm{s}}(\leq \pm 0.4)$ & $\tau(\mathrm{ps})(\leq \pm 0.3)$ & $\beta(\leq \pm 0.01)$ & $\varepsilon_{\infty}(\leq \pm 0.2)$ \\
\hline$x=0$ & & & & & $x=0.33$ & & & & \\
\hline $298 \mathrm{~K}$ & 78.89 & 8.25 & 1 & 5.2 & $298 \mathrm{~K}$ & 68.97 & 52.78 & 0.86 & 3.97 \\
\hline $303 \mathrm{~K}$ & 76.67 & 7.29 & 1 & 4.71 & $303 \mathrm{~K}$ & 67.3 & 46.53 & 0.86 & 3.73 \\
\hline $308 \mathrm{~K}$ & 74.65 & 6.48 & 1 & 4.21 & $308 \mathrm{~K}$ & 65.62 & 39.25 & 0.859 & 3.51 \\
\hline $313 \mathrm{~K}$ & 72.84 & 5.75 & 1 & 3.73 & $313 \mathrm{~K}$ & 64.71 & 34.52 & 0.865 & 3.53 \\
\hline $\begin{array}{l}318 \mathrm{~K} \\
x=0.01\end{array}$ & 70.64 & 5.17 & 1 & 3.25 & $\begin{array}{l}318 \mathrm{~K} \\
x=0.4\end{array}$ & 63.8 & 30.31 & 0.87 & 3.56 \\
\hline $298 \mathrm{~K}$ & 78.11 & 9.37 & 0.97 & 4.65 & $298 \mathrm{~K}$ & 66.98 & 53.94 & 0.865 & 4.55 \\
\hline $303 \mathrm{~K}$ & 75.94 & 8.28 & 0.981 & 4.24 & $303 \mathrm{~K}$ & 65.31 & 47.24 & 0.869 & 4.53 \\
\hline $308 \mathrm{~K}$ & 73.78 & 7.14 & 0.991 & 3.83 & $308 \mathrm{~K}$ & 63.63 & 40.12 & 0.867 & 4.51 \\
\hline $313 \mathrm{~K}$ & 71.69 & 6.46 & 0.991 & 3.4 & $313 \mathrm{~K}$ & 62.49 & 35.61 & 0.87 & 4.02 \\
\hline $\begin{array}{l}318 \mathrm{~K} \\
x=0.05\end{array}$ & 69.6 & 5.98 & 0.99 & 2.96 & $\begin{array}{l}318 \mathrm{~K} \\
x=0.6\end{array}$ & 61.34 & 31.26 & 0.87 & 3.52 \\
\hline $298 \mathrm{~K}$ & 78.02 & 14.65 & 0.882 & 4.26 & $298 \mathrm{~K}$ & 59.83 & 42.88 & 0.815 & 5.0 \\
\hline $303 \mathrm{~K}$ & 75.22 & 12.82 & 0.882 & 3.95 & $303 \mathrm{~K}$ & 58.52 & 38.13 & 0.818 & 4.68 \\
\hline $308 \mathrm{~K}$ & 72.43 & 11.44 & 0.884 & 3.65 & $308 \mathrm{~K}$ & 57.21 & 34.66 & 0.82 & 4.35 \\
\hline $313 \mathrm{~K}$ & 71.17 & 10.46 & 0.885 & 3.29 & $313 \mathrm{~K}$ & 56.25 & 31.37 & 0.824 & 4.01 \\
\hline $\begin{array}{l}318 \mathrm{~K} \\
x=0.1\end{array}$ & 69.92 & 9.50 & 0.886 & 2.94 & $\begin{array}{l}318 \mathrm{~K} \\
x=0.8\end{array}$ & 55.30 & 28.88 & 0.822 & 3.66 \\
\hline $298 \mathrm{~K}$ & 76.79 & 22.53 & 0.816 & 4.27 & $298 \mathrm{~K}$ & 53.51 & 30.95 & 0.798 & 3.8 \\
\hline $303 \mathrm{~K}$ & 74.52 & 19.97 & 0.814 & 3.77 & $303 \mathrm{~K}$ & 52.56 & 28.23 & 0.804 & 3.98 \\
\hline $308 \mathrm{~K}$ & 72.24 & 17.94 & 0.811 & 3.28 & $308 \mathrm{~K}$ & 51.62 & 25.48 & 0.809 & 4.14 \\
\hline $313 \mathrm{~K}$ & 70.52 & 16.42 & 0.819 & 3.06 & $313 \mathrm{~K}$ & 50.88 & 23.47 & 0.811 & 4.03 \\
\hline $\begin{array}{l}318 \mathrm{~K} \\
x=0.15\end{array}$ & 68.8 & 14.97 & 0.827 & 2.84 & $\begin{array}{l}318 \mathrm{~K} \\
x=0.9\end{array}$ & 50.13 & 22.08 & 0.813 & 3.9 \\
\hline $298 \mathrm{~K}$ & 75.16 & 31.98 & 0.81 & 3.91 & $298 \mathrm{~K}$ & 50.40 & 25.93 & 0.822 & 4.24 \\
\hline $303 \mathrm{~K}$ & 72.54 & 27.51 & 0.808 & 3.48 & $303 \mathrm{~K}$ & 49.77 & 23.43 & 0.824 & 4.16 \\
\hline $308 \mathrm{~K}$ & 69.93 & 24.53 & 0.805 & 3.05 & $308 \mathrm{~K}$ & 49.13 & 21.65 & 0.823 & 4.07 \\
\hline $313 \mathrm{~K}$ & 68.46 & 21.76 & 0.809 & 3.1 & $313 \mathrm{~K}$ & 48.48 & 20.12 & 0.826 & 3.9 \\
\hline $\begin{array}{l}318 \mathrm{~K} \\
x=0.2\end{array}$ & 66.98 & 19.18 & 0.813 & 3.15 & $\begin{array}{l}318 \mathrm{~K} \\
x=1\end{array}$ & 47.83 & 18.86 & 0.827 & 3.72 \\
\hline $298 \mathrm{~K}$ & 74.64 & 40.13 & 0.845 & 3.74 & $298 \mathrm{~K}$ & 47.29 & 20.92 & 0.895 & 4.45 \\
\hline $303 \mathrm{~K}$ & 72.26 & 34.64 & 0.848 & 3.76 & $303 \mathrm{~K}$ & 46.93 & 18.96 & 0.896 & 4.16 \\
\hline $308 \mathrm{~K}$ & 69.88 & 29.87 & 0.847 & 3.78 & $308 \mathrm{~K}$ & 46.64 & 17.81 & 0.896 & 3.86 \\
\hline $313 \mathrm{~K}$ & 68.05 & 26.13 & 0.851 & 3.63 & $313 \mathrm{~K}$ & 46.08 & 16.64 & 0.903 & 3.55 \\
\hline $318 \mathrm{~K}$ & 66.23 & 23.48 & 0.854 & 3.49 & $318 \mathrm{~K}$ & 45.53 & 15.63 & 0.91 & 3.23 \\
\hline
\end{tabular}

The dielectric parameters obtained from the dielectric relaxation spectra of mixtures through the Davidson-Cole function are collected in Table 1 and also shown in Figures 3 and 4. From Figure 3, the spread of the relaxation time $(\beta)$ sharply decreases when small amounts of DMSO are added to water or when a small amount of water is added to DMSO. On further addition of DMSO, a maximum value is obtained at $x_{\text {DMSO }}$ in

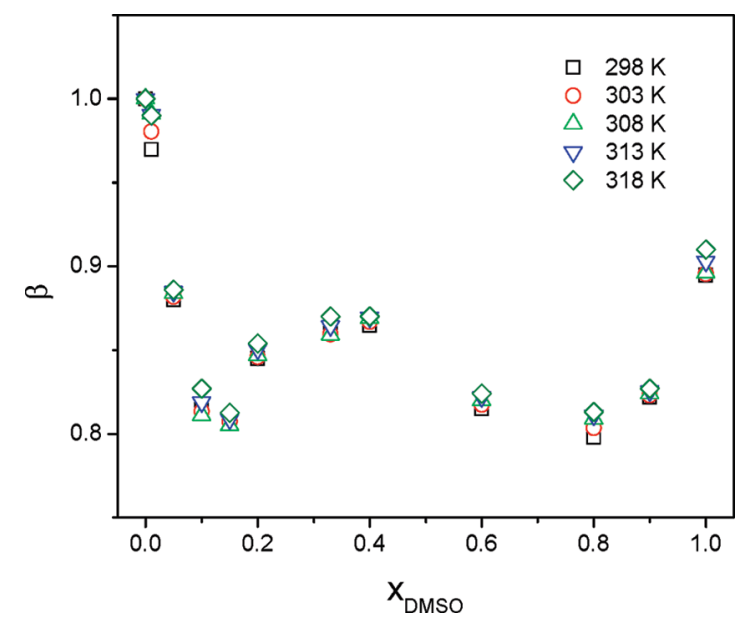

Figure 3. Plot of relaxation time distribution parameter $(\beta)$ of the Davidson-Cole function displayed as a function of mole fraction of DMSO for DMSO/water mixtures over the temperatures of 298-318 $\mathrm{K}$ at interval of $5 \mathrm{~K}$. the range of $0.3-0.4$. The change in $\beta$ values may reflect a variation in the relaxing species or a perturbation of the molecular structure of the system. The decrease of $\beta$ in the low DMSO concentration region suggests that the structure of the mixtures deviates significantly from that of pure water. The maximum $\beta$ at $x_{\mathrm{DMSO}}=0.3-0.4$ may indicate the formation of $\mathrm{DMSO} / 2 \mathrm{H}_{2} \mathrm{O}$ complexes, for which $x_{\text {DMSO }}=0.33$.

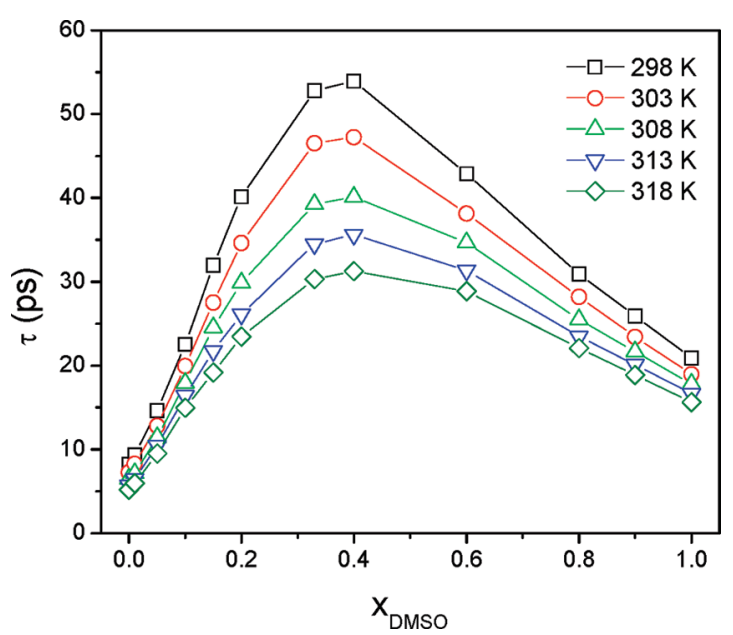

Figure 4. Plot of relaxation time $(\tau)$ of the Davidson-Cole function displayed as a function of mole fraction of DMSO for DMSO/water mixtures over the temperatures of $298-318 \mathrm{~K}$ at interval of $5 \mathrm{~K}$. 


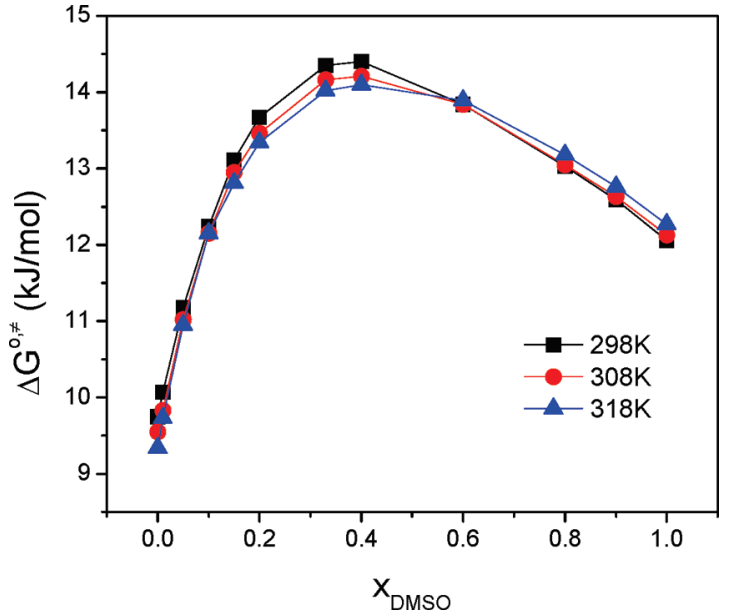

Figure 5. Free energy of activation $\left(\Delta G^{0, \ddagger}\right)$ for the dielectric relaxation process in the DMSO/water mixtures as a function of the solution composition at 298, 308, and $318 \mathrm{~K}$.

The dielectric relaxation time, $\tau$, as a function of the composition of the solution is shown in Figure 4. For each temperature, $\tau$ increases with $x_{\text {DMSO }}$ at small DMSO content, reaches a maximum when $x_{\text {DMSO }}$ is in the range of $0.3-0.4$, and then decreases to the pure DMSO value. Previous studies ${ }^{33,34}$ have attributed the increase in the relaxation time with DMSO concentration to hydrophobic hydration caused by the nonpolar methyl groups, in an analogy with hydrophobic hydration in macromolecules. However, this analogy ignores the effects of the strong hydrogen bonds between DMSO and water molecules. Therefore, the dielectric relaxation time information needs further analysis.

The dielectric relaxation can be treated as a rate process involving a path over a potential barrier. ${ }^{38,39,52,53}$ The energy of activation for the dielectric relaxation process, $\Delta G^{0, \mp}$, can be calculated from the dielectric relaxation time by using the Eyring equation

$$
\Delta G^{0, \ddagger}=R T \ln \left(\frac{k_{\mathrm{B}} T \tau}{h}\right)
$$

where $T$ is the absolute temperature, and $h, k_{\mathrm{B}}$, and $R$ are Plank's constant, Boltzmann's constant, and the molar gas constant, respectively. A plot of $\Delta G^{0, \ddagger}$ versus $T\left(\Delta G^{0, \ddagger}=\Delta H^{0, \ddagger}-T \Delta S^{0, \mp}\right)$ can yield the enthalpy $\Delta H^{0, \neq}$ and entropy $\Delta S^{0, \neq}$ of activation for the dielectric relaxation process. These parameters characterize the molecular interactions and dynamics of the components and their mixtures.

Figure 5 displays the free energy of activation as a function of composition at temperature of 298, 308, and $318 \mathrm{~K}$. From this figure, we can see that $\Delta G^{0, \ddagger}$ displays an extremely nonlinear dependence on DMSO mole fraction between 298 and $318 \mathrm{~K}$. These $\Delta G^{0, \ddagger}$ versus $x_{\text {DMSO }}$ curves indicate that there exist strong interactions between DMSO and water molecules, because, if the mixture was an ideal system, the free energy of activation of mixtures would have been represented by a straight line determined by $\Delta G_{\text {mix }}^{0, \neq}=\sum_{i} x_{i} \Delta G_{i}^{0, \neq}$, (where $x$ is the mole fraction of the species and the subscripts mix and $i$ represent the mixture and pure components, respectively).

Figure 6 shows that $\Delta G^{0, \ddagger}$ of DMSO/water mixtures is a linear function of temperature over the temperature range of 298-318 $\mathrm{K}$ for all compositions. The values of $\Delta H^{0, \ddagger}$ and $\Delta S^{0, \ddagger}$ obtained from this figure are displayed in Figure 7 as a function of

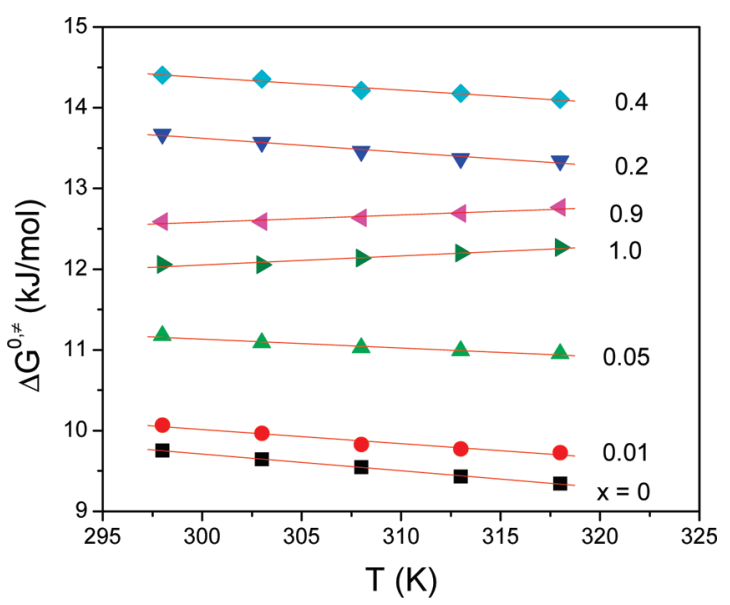

Figure 6. Free energy of activation $\left(\Delta G^{0, \ddagger}\right)$ for the dielectric relaxation process in the DMSO/water mixtures as a function temperature. The numbers in the figure denote the mole fractions of DMSO.

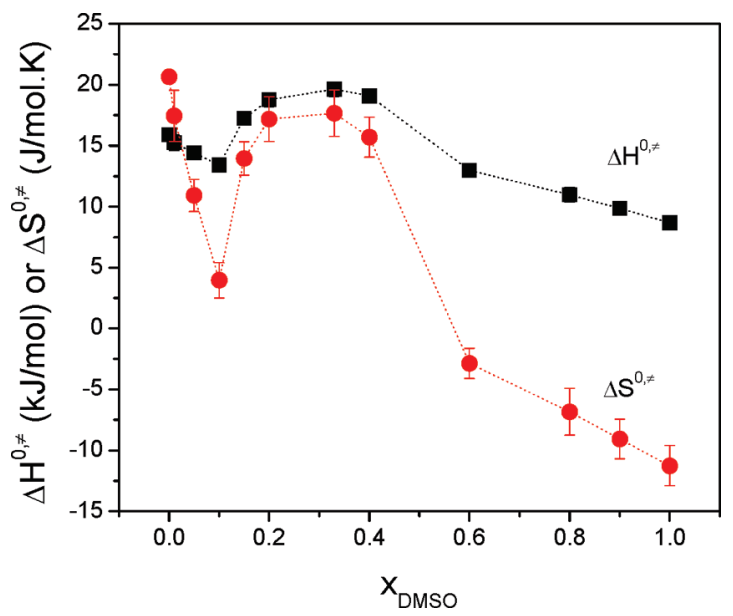

Figure 7. Concentration dependence of the enthalpy $\left(\Delta H^{0, \neq}\right)$ and the entropy $\left(\Delta S^{0, \neq}\right)$ of activation plotted against the molar fraction of DMSO.

mixture composition. For most mixtures, $\left|\Delta H^{0, \neq}\right|>\left|T \Delta S^{0, \neq}\right|$, indicating that the reorientation processes in pure liquids and mixtures are controlled by enthalpic rather than by entropic factors. Three different regions, separated by boundaries at $x_{\mathrm{DMSO}}$ $\sim 0.1$ and 0.6 , are apparent from the dependencies of $\Delta H^{0, \mp}$ and $\Delta S^{0, \neq}$ on composition, as shown in Figure 7. The following section will discuss the dielectric relaxation mechanism of the mixtures in each region.

\section{Discussion}

4.A. Low-DMSO Region $\left(0 \leq \boldsymbol{x}_{\text {DMSO }} \leq \boldsymbol{0 . 1}\right)$. The activation enthalpy $\left(\Delta H^{0, \mp}\right)$ and entropy $\left(\Delta S^{0, \neq}\right)$ for pure water are calculated to be $15.9 \mathrm{~kJ} / \mathrm{mol}$ and $20.7 \mathrm{~J} / \mathrm{mol} \cdot \mathrm{K}$, respectively. These values are in good agreement with data in the literature. ${ }^{34,38}$ Buchner et al. ${ }^{38}$ related the $\Delta H^{0, \mp}$ value to the $\mathrm{H}$-bond probability in water. Assuming that only water molecules with one H-bond are mobile, they estimated the average number of hydrogen bonds per water, $n_{\mathrm{HB}}$, as

$$
n_{\mathrm{HB}}=\frac{\Delta H^{0, \ddagger}}{\Delta H_{\mathrm{HB}}}+1
$$

where $\Delta H_{\mathrm{HB}}$ is the strength of the hydrogen bond, $\sim 10.9 \mathrm{~kJ} /$ mol. ${ }^{54}$ Adding 1 in eq 3 is based on the fact that these "mobile" 
TABLE 2: Activation Parameters $\left(\Delta H^{0, \neq}, \Delta S^{0, \neq}\right)$ and the Average Number of $\mathbf{H}$-Bonds That Must Be Broken during the Relaxation Process in the Temperature Range of 298-318 K

\begin{tabular}{lrrr}
\hline mole fraction & \multicolumn{1}{c}{$\Delta H^{0, \ddagger}, \mathrm{kJ} / \mathrm{mol}$} & $\Delta S^{0, \mp}, \mathrm{J} / \mathrm{mol} \cdot \mathrm{K}$ & $n_{\mathrm{HB}}$ \\
\hline 0 & $15.91 \pm 0.15$ & $20.67 \pm 0.40$ & 2.46 \\
0.01 & $15.25 \pm 0.45$ & $17.45 \pm 2.10$ & 2.42 \\
0.05 & $14.41 \pm 0.40$ & $10.92 \pm 1.30$ & 2.31 \\
0.1 & $13.40 \pm 0.45$ & $3.95 \pm 1.45$ & 2.23 \\
0.15 & $17.25 \pm 0.45$ & $13.94 \pm 1.40$ & \\
0.2 & $18.77 \pm 0.55$ & $17.18 \pm 1.85$ & \\
0.33 & $19.63 \pm 0.55$ & $17.66 \pm 1.90$ & \\
0.4 & $19.08 \pm 0.50$ & $15.70 \pm 1.65$ & \\
0.6 & $12.96 \pm 0.40$ & $-2.86 \pm 1.20$ & \\
0.8 & $10.98 \pm 0.50$ & $-6.83 \pm 1.90$ & \\
0.9 & $9.86 \pm 0.50$ & $-9.07 \pm 1.60$ & \\
1 & $8.67 \pm 0.50$ & $-11.26 \pm 1.65$ &
\end{tabular}

molecules usually have one intact hydrogen bond. Thus, a value of 2.5 is obtained for the average number of hydrogen bonds per water molecule in the liquid state at $298 \mathrm{~K}$, which is close to the literature value of $2.4,{ }^{55}$ obtained with molecular dynamics simulation, and of $2.8,{ }^{56}$ obtained from density data.

When a small amount of DMSO $(0 \leq x \leq 0.1)$ is added to water, $\Delta H^{0, \ddagger}$ and $\Delta S^{0, \ddagger}$ of the DMSO/water mixtures decrease from the values of pure water to a minimum at $x_{\mathrm{DMSO}}=0.1$ (Figure 7). The decrease of $\Delta H^{0, \ddagger}$ and $\Delta S^{0, \ddagger}$ in the mixtures can be attributed to two phenomena: a change in hydrogen-bond strength or a decrease in the average number of hydrogen bonds. IR spectra measurements ${ }^{10}$ show that the addition of DMSO did not change the distribution of hydrogen-bond energies of water. It is thus concluded that the reduction of $\Delta H^{0, \neq}$ and $\Delta S^{0, \neq}$ is due to the decrease of average number of hydrogen bonds. Applying the similar method in eq 3 the average numbers of $\mathrm{H}$-bonds were also calculated for the dilute solutions, with the results being summarized in Table 2. The reduction of $n_{\mathrm{HB}}$ in the mixtures means that the cooperative relaxation of the hydrogen-bond network is facilitated by the presence of DMSO. In other words, the DMSO molecules decrease the potential barrier for reorientation of the system dipole.

A hydrogen-bonding configuration has been proposed by Soper and Luzar ${ }^{13}$ to account for the neutron diffraction results. A similar configuration is redrawn in Figure 8, where a water molecule is replaced by a DMSO molecule. Due to the ability for DMSO to accept two hydrogen bonds, ${ }^{23}$ the short-range water structure remains in the presence of DMSO molecules. Owing to the steric constraints of two methyl groups, which prevent the further formation of hydrogen bonds on these sites, the total hydrogen bonds are decreased and the percentage of water molecules that are hydrogen-bonded to each other are substantially reduced compared to that in pure water (Figure 8). This allows interpreting the reduced $n_{\mathrm{HB}}$ values in the mixtures obtained from the dielectric measurement. Since DMSO forms stronger hydrogen bonds with water than water does with itself, ${ }^{6-8,57}$ the hydrogen bonding between water molecules is energetically more favorable to break or reform compared to the H-bond between DMSO and water. Thus, dielectric relaxation in DMSO/water mixtures is essentially the same process as it is in pure water, through the reorientation of the H-bonds between water molecules. The spatial steric constraints imposed by DMSO molecules on the hydrogen-bond network have at least two consequences: reducing the proportion of H-bonds that are energetically favorable and leading to longer waiting time for destabilized hydrogen to rejoin the network by forming new hydrogen bonds. Both of these two effects will

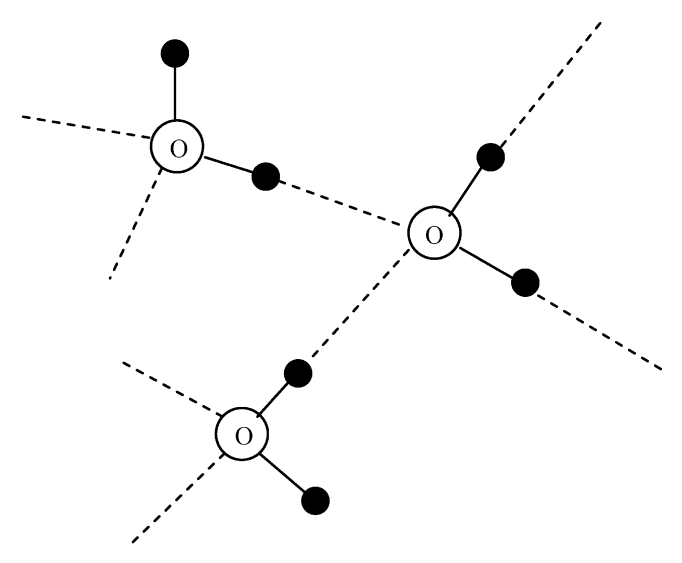

(a)

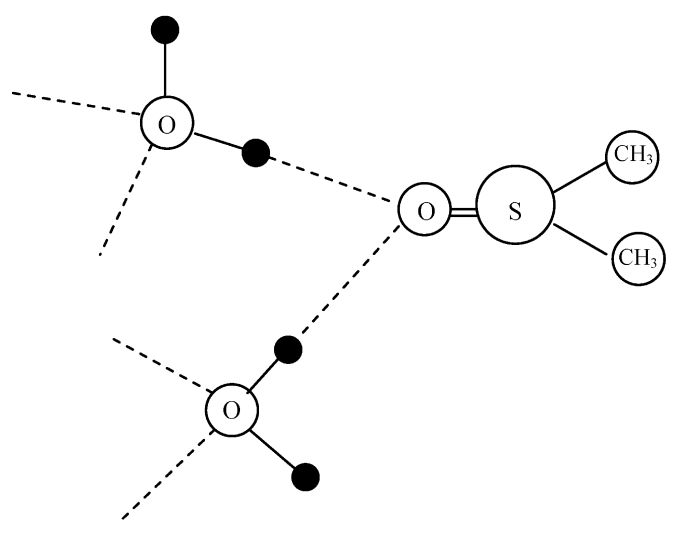

(b)

Figure 8. Schematic view of hydrogen bonding in pure water (a) and DMSO/water mixture (b). Solid lines represent intramolecular bonds; dashed lines represent hydrogen bonds.

result in higher relaxation times. This accounts for the increase in relaxation times in mixtures.

This structure-breaking effect due to the strong hydrogen bonding between DMSO and water is clearly manifested also in the dielectric measurements. This result is consistent with many other studies. ${ }^{10,19,23}$ Among them, the measurement of the temperature of maximum density provides a direct indication of water structure. Passage of the density through a maximum at $3.98{ }^{\circ} \mathrm{C}$ with increasing temperature is a unique property of water that can be attributed to a breakdown of the open hydrogen-bonded "flickering clusters" to form dense, nonstructured water, superimposed upon the increase in molar volume of both components as the temperature is raised. When a solute is added to water to form an ideal solution (one that does not have a specific impact on the structure of water) the temperature of maximum density $\left(T_{\mathrm{m}}\right)$ changes by an amount that is readily calculated from the properties of the system assuming ideal behavior. Subtraction of this change from the measured change in $T_{\mathrm{m}}$ yields a component that unequivocally reflects the impact of the solute on the unique structure of water. If the difference is positive, the solute buttresses the structure of water and is deemed to be a "structure maker"; if it is negative, the solute is a "structure breaker". DMSO at low concentration is found to be a water structure breaker. ${ }^{19}$ On the other hand, in a neutron and X-ray diffraction experiment, Stafford et al. ${ }^{7}$ observed an increase in the RDF (radial distribution function) intensities in dilute DMSO solutions and explained it as the promotion of 
the water structure by the presence of DMSO molecules. However, this increase may reflect mostly concentration changes rather than the influence of the second component. ${ }^{23}$ The contribution from nonpolar methyl groups, if there is any, may only appear in extremely dilute solutions.

4.B. Region $0.15<\boldsymbol{x}<\mathbf{0 . 4}$. As shown in Figure 7, the most outstanding feature for the dynamics of the mixtures in the concentration region of $0.15<x<0.4$ is that the activation enthalpy in this region is higher than that for pure water (Figure 7). $\Delta H^{0, \ddagger}$ reaches a maximum $(20 \mathrm{~kJ} / \mathrm{mol})$ at $x=0.33$, which is 1.3 times higher than that of pure water. This concentration also corresponds to the existence of minima or maxima in several thermodynamic properties. ${ }^{9-12}$ The explanation for this phenomenon lies in the hydrogen-bonding configuration in the mixtures. According to Figure 8, the fraction of $\mathrm{H}$-bond formed between DMSO and water molecules increases as the DMSO concentration increases, whereas the fraction of $\mathrm{H}$-bond between water molecules decreases. The ratio of these two types of $\mathrm{H}$-bonds will reach a maximum at a particular concentration. In view of the two hydrogen-bond accepting ability of the DMSO oxygen atom, the ratio will reach maximum at $x=0.33$, where the stoichiometric complex $\mathrm{H}_{2} \mathrm{O}-\mathrm{DMSO}-\mathrm{H}_{2} \mathrm{O}$ forms. At this concentration, each water molecule will be hydrogenbonded to a DMSO molecule, and the mixture will behave like a homogeneous liquid, as reflected by the maximum $\beta$ in Figure 3. The rotational relaxation of this mixture, without doubt, involves the hydrogen bond between DMSO and the water molecule. The higher activation enthalpies in the second region, in comparison with the bulk water, are then due to the change of the relaxation species, which have higher hydrogen-bond strengths. The maximum relaxation time in this region could be a result of the stronger hydrogen bonding and longer lifetime of these H-bonds.

The existence of stoichiometrically well-defined hydrogenbonded DMSO/water aggregates is suggested here based on the arguments of $\Delta H^{0, \neq}$ and $\Delta S^{0, \neq}$. Such aggregates are expected to add distinct contributions to the dielectric spectrum. However, there is no evidence of a distinct contribution to the dielectric relaxation in the spectra of these mixtures. This is not clearly understood. ${ }^{33}$ The explanation may be that the radii of the different aggregates in the system essentially have a continuous distribution. However, an alternative interpretation may involve the lifetimes of the different relaxation species. The relaxation times of aqueous DMSO solutions may reflect the production rate of certain mobile species, including mobile water and DMSO molecules.

4.C. Pure DMSO and DMSO-Rich Region $0.6<x \leq 1$. In sharp contrast to the above two regions, negative entropies of activation $\left(\Delta S^{0, \neq}\right)$ are obtained for the concentrated solutions, as seen in Figure 7. At the same time, the activation enthalpy decreases to less than the strength of a hydrogen bond (10.9 $\mathrm{kJ} / \mathrm{mol}$ ). In the concentrated solutions, the complete hydration of DMSO becomes impossible, because of the shortage of water molecules. The interaction between DMSO molecules is thus believed to contribute significantly to the properties in this region.

In pure DMSO liquid, a distinct antiparallel ordering of the molecular dipoles has been observed by static permittivity measurements ${ }^{33}$ and by molecular dynamics simulation. ${ }^{23}$

The dipole correlation, expressed by the Kirkwood correlation factor $g_{\mathrm{K}},{ }^{58}$ for pure DMSO can be calculated according to the Kirkwood-Frohlich equation ${ }^{32}$

$$
\varepsilon_{\mathrm{s}}-\varepsilon_{\infty}=\frac{3 \varepsilon_{\mathrm{s}}}{2 \varepsilon_{\mathrm{s}}+\varepsilon_{\infty}} \frac{4 \pi N}{3 k_{\mathrm{B}} T V} \frac{\left(\varepsilon_{\infty}+2\right)^{2} \mu^{2}}{9} g_{\mathrm{K}}
$$

where $\varepsilon_{\mathrm{s}}$ and $\varepsilon_{\infty}$ have the same meaning as above. $N$ is the number of particles, $V$ is the volume, and $\mu$ is the magnitude of the dipole moment. Taking the gas-state dipole moment, 3.91 $\mathrm{D},{ }^{25}$ for $\mu$, a value of 0.5 is obtained for $g_{\mathrm{K}}$ of DMSO at $25{ }^{\circ} \mathrm{C}$ from the permittivity data, indicating antiparallel ordering of neighboring dipoles. ${ }^{33}$ The dipolar interaction energy between neighboring dipoles can be further calculated as a rough approximation as

$$
E=\frac{\mu_{1} \mu_{2}}{4 \pi \varepsilon_{0} r^{3}}
$$

where $r$ is the distance between two neighboring DMSO molecules, which is approximately a molecular diameter. With a molecular diameter of $5.6 \mathrm{~A},{ }^{25}$ one obtains $E=6.4 \mathrm{~kJ} / \mathrm{mol}$ from eq 5 . The activation enthalpy determined by the dielectric measurements is close to $9 \mathrm{~kJ} / \mathrm{mol}$ (Table 2). Taking into account the oversimplification of the mean spherical molecule model, higher dipolar interaction energy is obtained in practice. It is thus concluded that antiparallel dipole-dipole interactions contribute significantly to the formation of local order in liquid DMSO.

The clusters of DMSO would be expected to exist in highly concentrated solutions due to the same dipolar interactions. In theory, the Kirkwood correlation parameter for the concentrated solutions could be also determined through eq 4 . However, the precise determination of $g_{\mathrm{K}}$ for the liquid mixtures is very difficult owing to the complex interaction between the components. As a rough approximation, the average dipole moment is taken as $\mu=\left(x_{\mathrm{w}} \mu_{\mathrm{w}}{ }^{2}+x_{\mathrm{DMSO}} \mu_{\mathrm{DMSO}}\right)^{1 / 2}$. The value of $g_{\mathrm{K}}$ for the DMSO/water mixtures can thus be calculated from eq 4 . Figure 9 shows the Kirkwood correlation factor $g_{\mathrm{K}}$ for the DMSO-rich mixtures at 25,35 , and $45^{\circ} \mathrm{C}$. $g_{\mathrm{K}}$ values of the mixtures are smaller than 1 , indicating the similar dipole correlation to that in pure DMSO. The significance of $g_{\mathrm{K}}$ for dilute solutions is less important than in concentrated solutions, due to the prevailing hydrogen bonding in dilute solutions, and is not shown in this figure. Obviously, antiparallel ordering is also significant in highly concentrated aqueous solutions.

\section{Conclusions}

The temperature dependence of the dielectric relaxation behavior at microwave frequencies of DMSO/water mixtures was investigated over the whole composition range. These mixtures exhibit different relaxation dynamics in different concentration regions, as revealed from an analysis of the relaxation time. At small DMSO mole fractions $\left(x_{\mathrm{DMSO}} \leq 0.10\right)$, both the activation enthalpy and entropy decrease from the pure water values to minimum values at $x_{\mathrm{DMSO}}=0.1$. The average number of hydrogen bonds per water molecule in the mixtures is calculated to decrease accordingly, assuming that the presence of a small amount of DMSO does not significantly change the strength of hydrogen bonding. A hydrogen-bonding configuration in DMSO/water mixtures is suggested, where a water molecule in a hydrogen-bonding network is replaced by a DMSO molecule. Through this configuration, the breakdown of water structure is introduced. The increase in the relaxation time in the mixtures does not appear to be due to the hydrophobic hydration of the methyl groups; instead it is due 


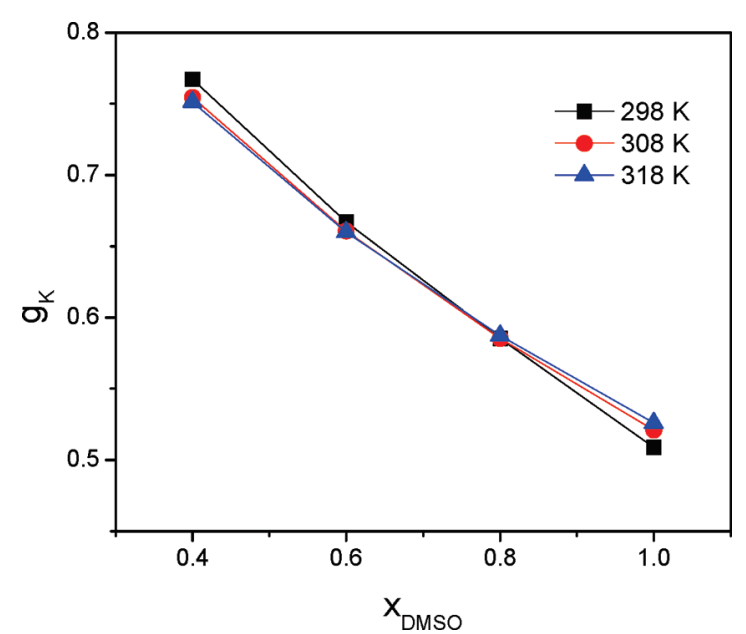

Figure 9. Kirkwood correlation factor $\left(g_{\mathrm{K}}\right)$ as a function of mole fraction of DMSO for the concentrated DMSO/water mixtures at 298, 308 , and $318 \mathrm{~K}$.

to the spatial steric constraints imposed by DMSO molecule on the hydrogen-bond network. At intermediate DMSO mole fractions $\left(0.15 \leq x_{\text {DMSO }} \leq 0.6\right)$, a stronger hydrogen bonding is suggested to form between DMSO and water molecules than between water molecules, based on the higher activation enthalpies in these mixtures than in pure water. The activation enthalpy and entropy reach maximum at the DMSO mole fraction of 0.33 . It seems likely that hydrogen-bonded $\mathrm{H}_{2} \mathrm{O}-\mathrm{DMSO}-\mathrm{H}_{2} \mathrm{O}$ complexes are formed in the mixtures. In the DMSO-rich region $\left(x_{\mathrm{DMSO}} \geq 0.6\right.$ ), the activation enthalpy is drastically decreased to less than the enthalpy of hydrogen bonding, and the activation entropy becomes negative. The negative activation entropy implies the self-association of DMSO molecules in these mixtures, such that the rotational activated complex is a more ordered structure than is the initial state. For neat DMSO liquid, an antiparallel arrangement of dipoles is obtained from the Kirkwood correlation factor, $g_{\mathrm{K}}=$ 0.5. The dipolar forces also contribute significantly to the dielectric relaxation of DMSO-rich mixtures.

Acknowledgment. Z.L. acknowledges Mr. Steve Perini and Mr. Khalid Rajab for help on dielectric measurements. The authors gratefully acknowledge financial support for this work by International Fuel Cells Inc. through subcontract no. 3540OB via contract no. DE-FCo4-02AL67608 and by the U.S. Department of Energy via contract no. DE-FG02-07ER46371 at Penn State University. E.M. additionally acknowledges support from the National Science Foundation (NSF Grant No. DMR0602877).

\section{References and Notes}

(1) Eisenberg, D.; Kauzmann, W. The Structure and Properties of Water; Oxford University Press: Oxford, 1969.

(2) Robinson, G. W.; Cho, C. H.; Urquidi, J. J. Chem. Phys. 1999, 111,698

(3) Chaplin, M. F. Biophys. Chem. 1999, 83, 211.

(4) Cho, C. H.; Singh, S.; Robinson, G. W. Faraday Discuss. 1996, $103,19$.

(5) Robsinson, G. W.; Zhu, S. B.; Singh, S.; Evans, M. W. Water in Biology, Chemistry and Physics; World Scientific Publishing Co. Ltd.: Singapore, 1996.

(6) Baker, E. S.; Jones, J. J. Phys. Chem. 1985, 98, 1730.

(7) Stafford, G. J.; Schaffer, P. C.; Leung, P. S.; Doebbler, G. F.; Brady, G. W.; Lyden, E. F. X. J. Chem. Phys. 1969, 50, 2140.

(8) Szmant, H. H. In Biological Actions of Dimethyl Sulfoxide; Jacob, S. W., Ed.; Annals of the New York Academy of Science, Vol. 243; Academic Press: New York, 1975.
(9) Cowie, J. M. G.; Toporowski, P. M. Can. J. Chem. 1964, 39, 2240.

(10) Fox, M. F.; Whittingham, K. P. J. Chem. Soc., Faraday Trans. $1975,71,1407$.

(11) Mazurkiewicz, J.; Tomasik, P. J. Phys. Org. Chem. 1990, 3, 493.

(12) Kaatze, U.; Brai, M.; Sholle, F. D.; Pottel, R. J. Mol. Liq. 1990, 44, 197.

(13) Soper, A. K.; Luzar, A. J. Chem. Phys. 1992, 97, 1320.

(14) Soper, A. K.; Luzar, A. J. Phys. Chem. 1996, 100, 1357.

(15) Luzar, A.; Soper, A. K.; Chandler, D. J. Chem. Phys. 1993, 99, 6836.

(16) Cabral, J. T.; Luzar, A.; Teixeira, J.; Bellissent-Funel, M. C. J. Chem. Phys. 2000, 113, 8736.

(17) Brink, G.; Falk, M. J. Mol. Struct. 1970, 5, 27.

(18) Lai, J. T. W.; Lau, F. W.; Robb, D.; Westh, P.; Nielsen, G.; Trandum, C.; Hvidt, A.; Koga, Y. J. Solution Chem. 1995, $24,89$.

(19) Macdonald, D. D.; Smith, M. D.; Hyne, J. B. Can. J. Chem. 1971, 49, 2817.

(20) Petrella, G.; Petrella, M.; Castagnolo, M.; Dell'Atti, A.; DeGiglio, A. J. Solution Chem. 1981, 10, 129.

(21) Mizuno, K.; Imafuji, S.; Ochi, T.; Ohta, T.; Maeda, S. J. Phys. Chem. B 2000, 104, 11001 .

(22) de Visser, C.; Henvelsland, W. J. M.; Dunn, L. A.; Somsen, G. J. Chem. Soc., Faraday Trans. 1978, 74, 1159.

(23) Vaisman, I. I.; Berkowitz, M. L. J. Am. Chem. Soc. 1992, 114, 7889

(24) Luzar, A.; Chandler, D. J. Chem. Phys. 1993, 98, 8160.

(25) Vishnyakov, A.; Lyubartsev, A. P.; Laaksonen, A. J. Phys. Chem. A 2001, 105, 1702 .

(26) Mancera, R. L.; Chalaris, M.; Samios, J. J. Mol. Liq. 2004, 110, 147.

(27) Chalaris, M.; Samios, J. J. Mol. Liq. 2002, 98-99, 399.

(28) Mancera, R. L.; Chalaris, M.; Refson, K.; Samios, J. Phys. Chem. Chem. Phys. 2004, 6, 94.

(29) Borin, I. A.; Skaf, M. S. J. Chem. Phys. 1999, 110, 6412.

(30) Skaf, M. S. J. Chem. Phys. 1997, 107, 7996.

(31) Zhang, Q.; Zhang, X.; Zhao, D. X. J. Mol. Liq. 2009, 145, 58; $145,67$.

(32) Scaife, B. K. P. Principles of Dielectrics; Clarendon Press: Oxford U.K., 1998.

(33) Kaatze, U.; Pottel, R.; Schafer, M. J. Phys. Chem. 1989, 93, 5623.

(34) Lyashchenko, A. K.; Lileev, A. S.; Novskova, T. A.; Kharkin, V. S. J. Mol. Liq. 2001, 93, 29.

(35) Achadov, J. J. Dielectric Properties of Binary Solutions; Pergamon: Oxford U.K., 1981.

(36) Puranik, S. M.; Kumbharkhane, A. C.; Mehrotra, S. C. J. Chem. Soc., Faraday Trans. 1992, 88, 433.

(37) Yang, L. J.; Yang, X. Q.; Huang, K. M.; Jia, G. Z.; Shang, H. Int. J. Mol. Sci. 2009, 10, 1261 57.

(38) Buchner, R.; Barthel, J.; Stauber, J. Chem. Phys. Lett. 1999, 306,

(39) Sato, T.; Chiba, A.; Nozaki, R. J. Mol. Liq. 2002, 101, 99; J. Chem. Phys. 1999, 110, 2508.

(40) Fini, G.; Mirone, P. Spectrochim. Acta 1976, 32A, 625.

(41) Sastry, M. I. S.; Singh, S. J. Raman Spectrosc. 1984, 15, 80.

(42) Czeslik, C.; Jonas, J. J. Phys. Chem. A 1999, 103, 3222.

(43) Lu, Z.; Polizos, G.; Macdonald, D. D.; Manias, E. J. Electrochem. Soc. 2008, 155, B163.

(44) Lu, Z.; Lanagan, M.; Manias, E.; Macdonald, D. D. J. Phys. Chem. $B$, 2009, DOI: $10.1021 /$ jp 9057115 .

(45) Havriliak, S.; Negami, S. Polymer 1967, 8, 161.

(46) Kaatze, U. J. Chem. Eng. Data 1989, 34, 371.

(47) Barthel, J.; Bachhuber, K.; Buchner, R.; Hetzenauer, H. Chem. Phys. Lett. 1990, 165, 369.

(48) Ellison, W. J. J. Phys. Chem. Ref. Data 2007, 36, 1.

(49) von Hippel, A. IEEE Trans. Electr. Insul. 1988, 23, 801.

(50) Barthel, J.; Buchner, R. Pure Appl. Chem. 1991, 63, 1473.

(51) Markarian, S. A.; Gabrielyan, L. S. Phys. Chem. Liq. 2009, 47, 311.

(52) Glasstone, S.; Laidle, L. J.; Eyring, H. The Theory of Rate Processes; McGraw-Hill: New York, 1941.

(53) Petong, P.; Pottel, R.; Kaatze, U. J. Phys. Chem. A 2000, 104, 7420.

(54) Walrafen, G. E.; Fisher, M. R.; Hokmabadi, M. S.; Yang, W. H.

J. Chem. Phys. 1985, 85, 6970.

(55) Zielkiewicz, J. J. Chem. Phys. 2005, 123, 104501.

(56) Stanley, H. E.; Teixeira, J. J. Chem. Phys. 1980, 73, 3404.

(57) Vishnyakov, A.; Lyubartsev, A. P.; Laaksonen, A. J. Phys. Chem. A 2001, 105, 1702.

(58) Bottcher, C. J. F. Theory of Electric Polarization; Elsevier Scientific: Amsterdam, The Netherlands, 1973; Vol. 1.

JP9059246 